\title{
BMJ Global Health Digital health vision: could MomConnect provide a pragmatic starting point for achieving universal health coverage in South Africa and elsewhere?
}

Garrett Livingston Mehl, ${ }^{1}$ Tigest Tamrat, ${ }^{1}$ Sanjana Bhardwaj, ${ }^{2}$ Sean Blaschke, ${ }^{3}$ Alain Labrique ${ }^{4}$

To cite: Mehl GL, Tamrat T, Bhardwaj S, et al. Digital health vision: could MomConnect provide a pragmatic starting point for achieving universa health coverage in South Africa and elsewhere?BMJ Glob Health 2018;3:e000626. doi:10.1136/ bmjgh-2017-000626

Received 25 January 2018 Accepted 2 March 2018

\section{Check for updates}

${ }^{1}$ Department of Reproductive Health and Research, World Health Organization, Geneva, Switzerland

${ }^{2}$ Unicef South Africa, Pretoria, South Africa

${ }^{3}$ Unicef, Eastern and Southern Africa Regional Office, Nairobi, Kenya

${ }^{4}$ Department of International Health, Johns Hopkins University Bloomberg School of Public Health, Baltimore, Maryland, USA

Correspondence to Dr Garrett Livingston Mehl; mehlg@who.int

\section{INTRODUCTION}

Digital health, the use of digital, mobile and wireless technologies for health, ${ }^{1}$ is being positioned as a transformative agent, particularly in low-income and middle-income settings, where mobile connectivity has reached unprecedented penetration and ubiquity. ${ }^{23}$ Multilateral initiatives such as the Roadmap for Health and Measurement and Accountability advocate the "use of the digital revolution to scale-up health interventions and engage civil society', and monitor health systems' performance and accountability to beneficiary populations. ${ }^{4}$ Over the past decade, numerous digital health strategies have emerged to address long-standing health system challenges, and achieve national and global goals including the United Nations Millenium Development Goals (MDGs) and United Nations Sustainable Development Goals (SDGs)..$^{56}$

Despite the global abundance of digital health implementations, few have achieved national-level scale or become institutionalised as routine practice within the Ministries of Health. Furthermore, successes are often defined as technical accomplishments, instead of demonstrated impact on programme or health outcomes. ${ }^{7}$ MomConnect, in South Africa, represents a rare example of a nationally scaled and government-supported digital health initiative. Core functionalities within MomConnect include unique registration of pregnant women; targeted communication in the form of stage-appropriate messages to pregnant and lactating women; and an interactive help desk for clients to provide feedback on the quality
Summary box

Targeted client communications such as text messages have emerged as a promising digital intervention to drive demand for services in low-income and middle-income country settings.

- MomConnect has achieved unprecedented coverage of pregnant women through registering over 1.5 million pregnant women, which over 3 years accounts for approximately $60 \%$ of this target population.

- MomConnect illustrates how an investment in the digital solutions within a particular health programmatic area (eg, maternal health) can simultaneously contribute towards the development of 'common good' foundational elements of an interoperable digital national health information system.

- Caution and rigour will be needed to ultimately ensure that these investments lead to positive impacts on maternal, newborn and child health, as well as reach even the most vulnerable populations.

of services received or seek further information. ${ }^{8}$ Women registered into MomConnect receive messages twice weekly-the content of which reflects the stage of pregnancy or postpartum period: the first encourages pregnant women to attend facilities and receive antenatal care (ANC); the second focuses on essential newborn care, nutrition including infant feeding, immunisations and hygiene. National and provincial campaigns were held to promote MomConnect, including promotion via radio, posters and through public sector health workers. Enrolment is contingent on access to a mobile phone, of which $89 \%$ of South Africans are estimated to have. Current estimates of smartphones 


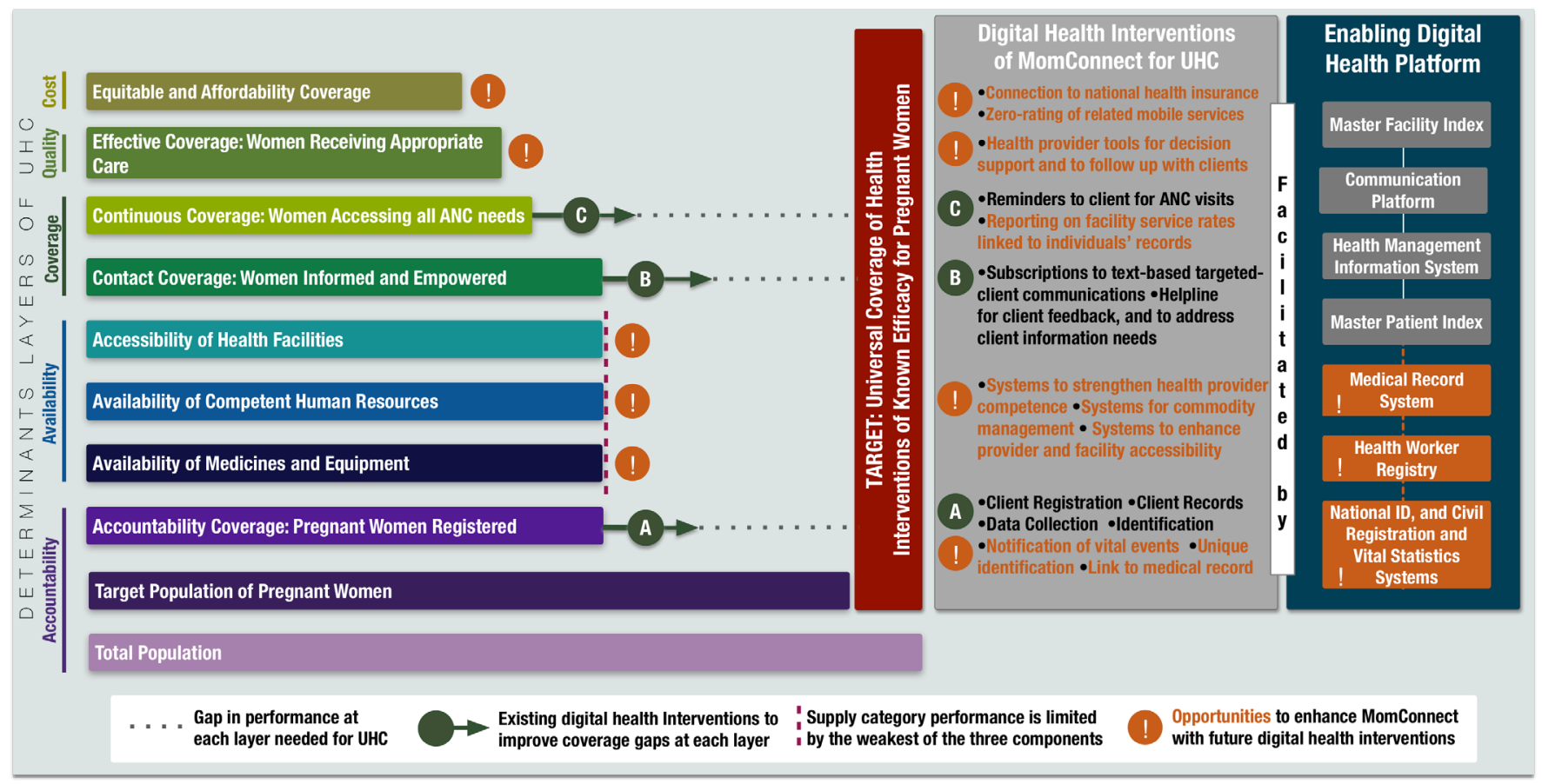

Figure 1 Enabling digital health architecture detailing digital health interventions in MomConnect for strengthening universal health coverage for pregnant women (adapted from ${ }^{18}$ ).

are $\sim 30 \%$. However, MomConnect registration was done via Unstructured Supplementary Service Data (USSD) to accommodate all phone types, and thus the type of phone is not deemed to be an impediment to enrolment/registration. Text messages are provided at no cost to registered users. As detailed in special supplement articles by Xiong et al and Engelhard et al, the help desk was used by just under $10 \%$ of registered MomConnect users, with women seeking information or wishing to lodge a complaint or compliment services received. ${ }^{9-11}$ Since its launch in 2014, MomConnect is one of the only digital health programmes in the world to have reached over $60 \%$ coverage of all pregnant women (calculated as proportion of women registered out of those attending ANC1) nationally, with 1.5 million subscribers registered. $^{1213}$

As countries try to identify pragmatic approaches to operationalise and achieve universal health coverage (UHC), many are augmenting their health systems with digital intervention, assuming future benefit will emerge. MomConnect provides evidence of the possible value of these investments. The coverage rates achieved by this programme in registering pregnant women speak to the transformative potential of mobile and digital technologies to achieve objectives difficult to reach using traditional methods. These gains towards universal pregnancy registration position MomConnect as an entrypoint to further pursue the UHC goals of ensuring that everyone has access to affordable, essential, high-quality services, where and when they need them, beginning with this important cohort of pregnant women. In this paper, we examine strides made by MomConnect to expand access to health information and explore the opportunities to harness this momentum to further strengthen health systems and achieve health outcomes.

\section{WHAT MOMCONNECT OFFERS}

South Africa has one of the highest mobile penetration rates in the world, with a 2016 mobile-cellular subscription rate of $142 \% .{ }^{14}$ The South African National Department of Health $(\mathrm{NDoH})$ capitalised on this widespread use of mobile technology when it launched MomConnect to equip pregnant women with critical health information in the effort to tackle maternal and infant mortality. At its inception in 2014, the NDoH set a target of reaching 1.2 million pregnant women across South Africa. ${ }^{15}$ Three years later, MomConnect has cumulatively registered over 1.5 million unique subscribers (ie, pregnant and lactating women) across approximately 4700 health facilities nationally. ${ }^{8}$ These numbers represent a remarkable achievement towards a goal of universal registration of pregnant women in South Africa. Furthermore, it demonstrates the thoughtful design to ensure broad population accessibility, while also providing a hopeful illustration that access to information should no longer be the privilege of an elite population subgroup. Key elements of a planned digital health implementation are summarised below as possible guideposts to consider in planning future digital investments in South Africa and elsewhere.

\section{A NATIONAL VISION AND ARCHITECTURE FOR DIGITAL HEALTH}

MomConnect was established as a core registration and messaging service of a planned national digital 
architecture. There was considerable forethought to how the investment in MomConnect would be leveraged in a staggered manner as an initial investment towards an interoperable national health information system. MomConnect is situated within a technical architecture that includes a master patient index (Open Enterprise Patient Index), medical record system (ie, OpenMRS), master facility index and health management information system (ie, District Health Information System 2), along with an interoperability layer for exchanging data between the various systems. ${ }^{16}$ This extensive investment in the digital health architecture prior to deploying MomConnect laid the ground for future capabilities. Furthermore, MomConnect shifted an existing paradigm of targeting messages to a group of people, with only knowledge of their phone number, to one where clients were formally registered by health workers, allowing both virtual and inperson services to be delivered to them. System interoperability allows MomConnect to serve as a component of a health service package that combines both digital and face-to-face services.

\section{MOVING FROM UNIVERSAL PREGNANCY REGISTRATION TO UHC}

UHC serves as a guiding principle within the UN SDGs to ensure all individuals 'receive the health services they need, of sufficient quality to be effective while at the same time ensuring that the use of these services does not expose the user to financial hardship'. ${ }^{17}$ Although MomConnect has aggressively worked towards universal pregnancy registration, it is also important to ensure that the important objectives of coverage, quality and equity are met to arrive closer to the goals of UHC. Below we have leveraged Mehl's 2014 cascading health systems model to illustrate how MomConnect begins to address fundamental UHC priorities among pregnant women (figure 1). ${ }^{18}$ The model illustrates how health systems lose performance to reach target populations with essential health interventions because of 'bottlenecks' at successive levels, each dependent on the previous layer. For a particular target population, gaps in information, training, quality and equitable distribution contribute to accumulated losses in potential, leading to diminished health system performance. The model helps illustrate opportunities for improvements at particular strata of the system, with cascading benefits to each layer above it. Specifically, when applied to pregnant women targeted by MomConnect, the model suggests the following necessary health system responsibilities: to identify all women who are pregnant and register them; ensure they have access to facilities and to competent providers; and that commodities necessary to provide antenatal and postnatal services are in stock. The UHC framework applied to pregnancy suggests that women receive information appropriate to their stage of pregnancy; that women access facilities at the appropriate times; and that providers effectively provide essential and appropriate services throughout their pregnancy and childbirth. This also implies that for the women and their families, these services should be affordable and equitable, and the experience of pregnancy and childbirth should not expose them to financial hardship.

To achieve UHC, it is important to ensure that the complete triad of coverage, quality and equity is met. Among the foundational challenges of achieving UHC in any context, especially low-resource settings, is the identification of a precise and complete denominatorthat is, the careful definition of the population eligible for and entitled to particular services (eg, target population). Strengthening denominators is also an important component of reducing inequities in the reach of health services to the proverbial 'last mile'. MomConnect's central feature of supporting universal pregnancy registration facilitates this task at a population level. By virtue of its linkage to broader national systems (figure 1), including the 'master patient index', women identified by MomConnect can be connected to other services and domains, improving coverage rates across the health system.

Once women are registered with MomConnect (figure 1A), the system leverages digital targeted client communication to deliver via text message to inform and empower pregnant women with content appropriate to their stage of pregnancy or postnatal child caretaking. These demand generation messages inform, empower and encourage appropriate client health behaviours including timely ANC, nutrition practices and facility-based care seeking (figure 1B,C). Women who have questions or needs, or who wish to provide feedback on their experience with the health system, may access the MomConnect help desk (figure 1C). This approach facilitates system feedback on quality of care, while also informing decision-makers of successes or lapses in service provision. Notably, MomConnect increases equity of access by forgoing technological sophistication, using digital approaches that have no cost to users.

\section{OPPORTUNITIES TO ENHANCE MOMCONNECT}

Within the cascading framework, MomConnect currently facilitates establishment of the target population and uses messages to generate initial demand for services-contact coverage. Reminders to pregnant women encourage appropriate health behaviour and timely use of ANC services, continuous coverage. ${ }^{18}$ While digital interventions provided through MomConnect clearly contribute to different determinant layers of UHC, there remain considerable opportunities for future enhancement, which would further benefit from additional integration with existing systems in the evolving South African national digital health architecture.

While the help desk feature of MomConnect contributes towards monitoring of quality of care, it does not link the pregnancy registration to monitoring health outcomes nor does it account for the receipt of services. 
Being able to track clinical outcomes both for the mother and the baby would be an important area to consider going forward, which the existing efforts to establish an interoperable digital health architecture could further facilitate.

In due time, there will be considerable value in strengthening the unique client identification approach and linking MomConnect data with medical record systems. These functionalities would contribute a standardised client record and support continuity of care along the care continuum from pregnancy to delivery to postnatal care, for the mother and the child. These integrations would prove valuable for monitoring individual women's services received or missed, which involve several 'touch points' across facilities including ANC services, delivery, immunisation, birth registration and social protection programmes. Additionally, should MomConnect be linked to a medical record used across facilities, it becomes possible to monitor which interventions (in the form of messages or reminders) affect service utilisation, health outcomes and perceived service experience of individual mothers. Further integration with a Health Worker Registry would facilitate understanding interactions between individual mothers and health providers, and offer insight into specific worker performance and association with health outcomes. Lastly, MomConnect integration with national ID and civil registration and vital statistics systems to contribute birth and mortality events would additionally provide valuable information about accountability and equity of the health system to beneficiary populations.

\section{MOMCONNECT DEMONSTRATES AN EXTENSIBLE AND MODULAR DIGITAL APPROACH FOR UHC GOALS ACROSS AFRICA}

As a complement to the 2016 District Planning Guidelines, the Ugandan Ministry of Health has invested in 'Family Connect', which draws heavily from, and extends, South Africa's MomConnect approach. In Uganda, Family Connect includes client registration and messaging, and notably extends the approach through digital systems integrations. Health Management Information System (HMIS) integration supports monitoring UHC at the district level, while linkages with the health workforce registry and medical record system support provider performance and client outcome tracking. The replication of MomConnect suggests the approach resonates with other countries' UHC goals. Furthermore, Uganda has demonstrated that the core functionality of MomConnect can be further customised to other countries' needs through customisation and appropriate systems integrations.

\section{A PRAGMATIC AND INCREMENTAL APPROACH TOWARDS UHC}

MomConnect has demonstrated how systematic and thoughtful digital health investments can serve as a gateway to improve UHC. Alongside strong political support for a programme that made basic pregnancy and health information available to and accessible by most of its citizens, South Africa also made systematic investments into other, less visible, components of digital architecture. By leveraging opportunities to extend the basic model of 'face-to-face client communication', the MomConnect programme added features that translated into real values for the health system. A humble text messaging programme soon became an important channel for extending the known denominator of pregnancies, for stimulating demand for brick-and-mortar services in facilities and to create feedback loops that stimulated improvements in quality of care.

The value potential of MomConnect has not been fully exhausted, but offers future opportunities for a health system that is responsive and programmes that can adjust according to immediately identified gaps and needs. However, the successful realisation of this vision will require active use of data and triangulation of information across multiple systems, sustained commitment and resourcing, and continuous tracking of outcomes to ensure positive effects on maternal and newborn care.

Despite the achievements and promises of MomConnect, evidence of the impact of this initiative is still nascent. Rigorous efforts are needed to demonstrate the effect on maternal, newborn and child health outcomes, and ensure vulnerable populations are not unduly burdened or left behind. MomConnect has demonstrated, unequivocally, that large-scale, integrated digital health strategies are no longer a future pipe dream, but that such systems are feasible. The programme has demonstrated the value in investing in the enabling architecture for scaling digital solutions-even when the fruit of such investments may not be immediately apparent. The unhurried, strategic vision which the South African government continues to deploy might well serve as an example to digital innovators everywhere-reminding us that a strong foundation and clear blueprint is more important for long-term success than a shiny facade, especially as we consider digital investments seriously to accelerate our course towards UHC.

Acknowledgements The support provided by John Snow, Inc. (JSI) in the President's Emergency Plan for AIDS Relief (PEPFAR) and United States Agency for International Development (USAID)-funded MEASURE Evaluation Strategic Information for South Africa (MEval-SIFSA) project to enable this publication is acknowledged with gratitude.

Contributors GLM, TT, SaB, SeB and AL contributed to the conception, drafting and revision of the manuscript, approved the final manuscript submission, and have agreed to the accuracy of the content.

Funding The authors have not declared a specific grant for this research from any funding agency in the public, commercial or not-for-profit sectors.

Competing interests None declared.

Patient consent Not required.

Provenance and peer review Not commissioned; externally peer reviewed.

Data sharing statement No additional data are available.

Open Access This is an Open Access article distributed in accordance with the Creative Commons Attribution Non Commercial (CC BY-NC 4.0) license, which permits others to distribute, remix, adapt, build upon this work non-commercially, 
and license their derivative works on different terms, provided the original work is properly cited and the use is non-commercial. See: http://creativecommons.org/ licenses/by-nc/4.0/

(C) Article author(s) (or their employer(s) unless otherwise stated in the text of the article) 2018. All rights reserved. No commercial use is permitted unless otherwise expressly granted.

\section{REFERENCES}

1. World Health Organization. Monitoring and evaluating digital health interventions: a practical guide to conducting research and assessment. Geneva: World Health Organization, 2016.

2. International Telecommunication Union. ITU releases 2015 ICT figures. http://www.itu.int/net/pressoffice/press_releases/2015/17. aspx\#.Wd883GmGPRY (accessed 10 Oct 2017).

3. GSMA Intelligence. Global mobile trends. www.gsmaintelligence. comhttps://www.gsmaintelligence.com/research/?file=357f1541c77 358e61787fac35259dc92\&download (cited 24 Feb 2018).

4. World Bank, United States Agency for International Development, World Health Organization. The roadmap for health measurement and accountability. http://www.who.int/hrh/documents/ roadmap4health-measurement_accountability.pdf?ua $=1$

5. Labrique $A B$, Vasudevan L, Kochi E, et al. mHealth innovations as health system strengthening tools: 12 common applications and a visual framework. Glob J Health Sci 2013;1:160-71.

6. Mehl G, Vasudevan L, Gonsalves L, et al. Harnessing mHealthin lowresource settings to overcome health system constraints andachieve universal access to healthcare. Behavioral health care and technology: using science-based innovations to transform practice. 2014.

7. Huang F, Blaschke S, Lucas H. Beyond pilotitis: taking digital health interventions to the national level in China and Uganda. Global Health 2017;13:49.
8. National Department of Health. What is momconnect. http://www. health.gov.za/index.php/mom-connect (accessed 10 Oct 2017).

9. Xiong K, Kamunyori J, Sebidi J. The MomConnect help desk: how an interactive mobile messaging programme is used by mothers in South Africa. BMJ Global Health 2018;3(Suppl 2):e000578.

10. Engelhard MM, Copley C, Watson J, et al. Optimising mHealth helpdesk responsiveness in South Africa: towards automated message triage. BMJ Global Health 2018;3(Suppl 2):e000567.

11. Skinner D, Delobelle P, Pappin M, et al. User assessments and the use of information from MomConnect, a mobile phone text-based information service, by pregnant women and new mothers in South Africa. BMJ Global Health 2018;3(Suppl 2):e000561.

12. Peter J, Benjamin P, LeFevre AE, et al. Taking digital health innovation to scale in South Africa: ten lessons from MomConnect. BMJ Global Health 2018;3(Suppl 2):e000592.

13. Lefevre AE, Dane $P$, Copley $C$, et al. Unpacking the performance of a mobile health information messaging program for mothers (MomConnect) in South Africa: evidence on program reach and messaging exposure. BMJ Global Health 2018;3(Suppl 2):e000583.

14. International Telecommunication Union. South Africa profile. 2016 https://www.itu.int/net4/itu-d/icteye/CountryProfileReport.aspx? countrylD=7 (accessed 10 Oct 2017).

15. United Nations Foundation Innovation Working Group. MomConnect: launching a national digital health program in South Africa. http://www.unfoundation.org/assets/pdf/momconnect-casestudy.pdf (accessed 10 Oct 2017).

16. Seebregts $C$, Dane $P$, Parsons AN, et al. Designing for scale: optimising the health information system architecture for mobile maternal health messaging in South Africa (MomConnect). BMJ Global Health 2018;3(Suppl 2):e000563.

17. United Nations. UN Sustainable Development Goals. https://www.un.org/sustainabledevelopment/health/ (accessed 15 March 2018).

18. Mehl G, Labrique A. Prioritizing integrated $m$ Health strategies for universal health coverage. Science 2014;345:1284-7. 